\title{
Comment on: Non-vitamin K Antagonist Oral Anticoagulants Versus Warfarin in Patients with Atrial Fibrillation and Liver Disease
}

\author{
Igor Vaz ${ }^{1} \oplus \cdot$ Ashish Kumar $^{2} \cdot$ Mariam Shariff $^{2} \cdot$ Rajkumar Doshi $^{3}$
}

Published online: 11 August 2020

C) Springer Nature Switzerland AG 2020

Sir,

We read with interest the systemic review and meta-analysis by Fu et al. [1] published in the American Journal of Cardiovascular Drugs. We have the following concerns. From the meta-analysis, the authors concluded that non-vitamin $\mathrm{K}$ antagonist oral anticoagulants (NOACs) were associated with a lower risk of all-cause mortality and major bleeding than warfarin in patients with atrial fibrillation and liver disease. However, as interpreted with Higgins $I^{2}$ statistics, these pooled estimates were associated with considerable heterogeneity. This could be because of the difference in the severity of liver disease among included studies. All the currently approved NOACs undergo significant hepatic metabolism, and their use in liver disease is not well-documented. While NOACs can be used in patients with mild to moderate liver disease (Child-Pugh A and B), warfarin is the only recommended oral anticoagulant in patients with severe liver disease (Child-Pugh C) [2]. The authors also concluded that
NOACs were noninferior to warfarin in patients with atrial fibrillation and liver disease, but the present meta-analysis did not define a noninferiority margin.

\section{Compliance with Ethical Standards}

Conflict of Interest Igor Vaz, Ashish Kumar, Mariam Shariff, and Rajkumar Doshi have no potential conflicts of interest that might be relevant to this Letter to the Editor.

\section{References}

1. Fu Y, Zhu W, Zhou Y, Chen H, Yan L, He W. Non-vitamin K antagonist oral anticoagulants versus warfarin in patients with atrial fibrillation and liver disease: a meta-analysis and systematic review. Am J Cardiovasc Drugs. 2020;20:139-47. https://doi. org/10.1007/s40256-019-00369-x.

2. Qamar A, Vaduganathan M, Greenberger NJ, Giugliano RP. Oral anticoagulation in patients with liver disease. J Am Coll Cardiol. 2018;71:2162-75.

This comment refers to the article available at https://doi. org/10.1007/s40256-019-00369-x.

An author's reply to this comment is available at https://doi. org/10.1007/s40256-020-00413-1

Igor Vaz

igorvaz@gmail.com

1 Department of Internal Medicine, Jackson Memorial Hospital/University of Miami, Miami, USA

2 Department of Critical Care Medicine, St John's Medical College Hospital, Bangalore, Karnataka, India

3 Department of Internal Medicine, University of Nevada Reno School of Medicine, Reno, Nevada, USA 